\title{
Numerical Analysis of an Adhesive Contact Problem with Long Memory
}

\author{
Xiaoliang Cheng and Qichang Xiao* \\ School of Mathematical Sciences, Zhejiang University, \\ Hangzhou 310027, P.R. China.
}

Received 18 October 2018; Accepted (in revised version) 2 April 2019.

\begin{abstract}
Spatially semidiscrete and fully discrete schemes for a variational-hemivariational inequality, which describes adhesive contact between a deformable body of a viscoelastic material with long memory and a foundation are constructed. The variational formulation of the problem is represented by a system coupling a nonlinear integral equation with a history-dependent variational-hemivariational inequality. Assuming certain regularity of the solution and using piecewise linear finite element function for displacements and piecewise constant functions for bonding field, we obtain optimal order error estimates.
\end{abstract}

AMS subject classifications: 65M10, 78A48

Key words: Variational-hemivariational inequality, adhesion, memory term, numerical approximation, error estimate.

\section{Introduction}

The mathematical theory of hemivariational inequalities plays an important role in a variety of subjects, ranging from nonsmooth mechanics, physics, and engineering to economics. As the result, a large number of mechanical contact problems lead to mathematical models expressed in terms of hemivariational inequalities. In recent years, the mathematical theory devoted to this field grows rapidly. Several results on hemivariational inequalities can be found in $[9,13-15]$.

In spite of vast literature on the modeling of contact problems and associated hemivariational inequalities, numerical methods for the problems with adhesion are not well developed. In this work, we deal with the numerical analysis of an adhesive contact problem for viscoelastic materials with long memory. Such models are closely connected to variational-hemivariational inequalities.

Let us recall the contact problem studied in [7]. Suppose that a viscoelastic body occupies an open bounded connected set $\Omega$ in the space $\mathbb{R}^{d}$, where $d=2$ or $d=3$. The Lipschitz ${ }^{*}$ Corresponding author. Email addresses: xiaoliangcheng@zju.edu.cn (X. Cheng), xiao_qc@126.com (Q. Xiao) 
continuous boundary $\Gamma$ of $\Omega$ consists of three mutually disjoint measurable sets $\Gamma_{D}, \Gamma_{N}$ and $\Gamma_{C}$ such that the $(d-1)$-dimensional Hausdorff measure of $\Gamma_{D}$ is positive. We are interested in the evolution of the mechanical state of the body on a finite time interval $[0, T]$. This evolution is caused by volume forces of density $f_{0}$ in $\Omega$ and by surface tractions of density $f_{N}$ on $\Gamma_{N}$. Besides, the viscoelastic body is in contact with another body called foundation or obstacle over the surface $\Gamma_{C}$. The surface $\Gamma_{C}$ is referred to as the contact surface. We introduce the following notation. Let $\mathbb{S}^{d}=\mathbb{R}_{s y m}^{d \times d}$ be a space of symmetric $d \times d$ real matrices and

$$
Q=\Omega \times(0, T), \quad \Sigma_{D}=\Gamma_{D} \times(0, T), \quad \Sigma_{N}=\Gamma_{N} \times(0, T), \quad \Sigma_{C}=\Gamma_{C} \times(0, T) .
$$

The classical formulation of the problem is as follows.

Problem 1.1. Find a displacement field $\boldsymbol{u}: Q \rightarrow \mathbb{R}^{d}$ and a stress field $\sigma: Q \rightarrow \mathbb{S}^{d}$ such that

$$
\begin{array}{ll}
\boldsymbol{\sigma}(t)=\mathscr{A}(t, \boldsymbol{\varepsilon}(\boldsymbol{u}(t)))+\int_{0}^{t} \mathscr{R}(t-s) \boldsymbol{\varepsilon}(\boldsymbol{u}(s)) d s & \text { in } \Omega, \\
\operatorname{Div} \boldsymbol{\sigma}(t)+\boldsymbol{f}_{0}(t)=\mathbf{0} & \text { in } \Omega, \\
\boldsymbol{u}(t)=\mathbf{0} & \text { on } \Sigma_{D}, \\
\boldsymbol{\sigma}(t) \boldsymbol{v}=\boldsymbol{f}_{N}(t) & \text { on } \Sigma_{N}, \\
\begin{cases}u_{v}(t) \leq g, \sigma_{v}(t)+p_{v}\left(\beta(t), u_{v}(t)\right) \leq 0, & \text { on } \Sigma_{C}, \\
\left(u_{v}(t)-g\right)\left(\sigma_{v}(t)+p_{v}\left(\beta(t), u_{v}(t)\right)\right)=0 & \text { on } \Sigma_{C}, \\
-\boldsymbol{\sigma}_{\tau}(t) \in \partial j_{\tau}\left(\beta(t), \boldsymbol{u}_{\tau}(t)\right) & \text { on } \Sigma_{C}, \\
\dot{\beta}(t)=F\left(u_{v}(t), \boldsymbol{u}_{\tau}(t), \beta(t)\right) & \text { on } \Sigma_{C}\end{cases} \\
\beta(0)=\beta_{0} &
\end{array}
$$

for all $t \in(0, T)$.

Here and in what follows, $\boldsymbol{\varepsilon}(\boldsymbol{u})$ denotes the linearised (small) strain tensor with components $\boldsymbol{\varepsilon}_{i j}(\boldsymbol{u})=(\boldsymbol{\varepsilon}(\boldsymbol{u}))_{i j}=\left(u_{i, j}+u_{j, i}\right) / 2$ and $u_{i, j}=\partial u_{i} / \partial x_{j}$. Moreover, Div $\boldsymbol{\sigma}=\left(\sum_{j=1}^{d} \sigma_{i j, j}\right)$ stands for the divergence of the stress tensor $\sigma$ and the dot above a variable represents time derivative. For simplicity, we do not show the dependence of various functions on variables $x$ and $t$ explicitly. The Eq. (1.1) represents the viscoelastic constitutive law with an elasticity operator $\mathscr{A}$ and a relaxation operator $\mathscr{R},(1.2)$ is the equilibrium equation and (1.3) and (1.4) are the displacement and the traction boundary conditions, respectively.

The contact condition (1.5) without adhesion was first introduced in [10]. It shows that the contact follows a normal compliance condition with the adhesion

$$
\sigma_{v}(t)=-p_{v}\left(\beta(t), u_{v}(t)\right)
$$

up to the limit $g$ and if the limit is reached, the contact follows the Signorini-type unilateral condition with the gap $g$. 
Condition (1.6) describes the friction law with adhesion, where $j_{\tau}$ is a given function and $\partial j_{\tau}$ denotes the Clarke subdifferential of $j_{\tau}$ with respect to the last variable. Various frictional conditions, which produce the subdifferential boundary conditions of the form (1.6) with a function $j_{\tau}$ satisfying the assumption $H\left(j_{\tau}\right)$ below, can be found in $[11,13]$. The examples include the nonmonotone friction law, the Tresca friction law and the powerlaw friction. Finally, the Eq. (1.7) models the evolution of the bonding field, (1.8) is the initial condition and $\beta_{0}$ the initial bonding field.

Problem 1.1 has been studied in [7], where an abstract variational formulation contact models is proposed. It consists of a variational-hemivariational inequality for the displacement field and an ordinary differential equation for the bonding field. The existence and uniqueness of a weak solution is proved in [7] by using a surjectivity result from [14]. Here, we continue the studies [7] and obtain error estimates for semidiscrete and fully discrete schemes.

Contact models with adhesion are also considered in $[2,5,6,12,16-20]$, where quasistatic and dynamic adhesive contact problem with subdifferential frictional boundary conditions and normal compliance with and without unilateral constraints have been studied. Numerical methods for such models are discussed in [19] with emphasis on spatially semidiscrete and fully discrete approximations for frictionless contact problems with adhesion. A static problem representing a simplified model of unilateral adhesive contact is considered in [6], with Galerkin and finite element methods used for solving a stationary variational-hemivariational inequality.

The aim of this work is to present the numerical analysis of spatially semidiscrete and fully discrete schemes for a quasistatic history-dependent contact problem with adhesion. The bonding field and the adhesive boundary contact on the tangential plane are described by an abstract ordinary differential equation and abstract subdifferential condition for a nonconvex function, respectively. Therefore, error estimates for displacements and bonding fields are valid for a variety of history-dependent contact problems with adhesion.

The paper is organised as follows. In Section 2, we provide preliminary information and list the main assumptions on the data and also construct an associated history-dependent variational-hemivariational inequality and recall the existence and uniqueness result from [7]. Section 3 deals with a spatially semidiscrete scheme for the inequality mentioned and the error estimates. In Section 4, we develop a fully discrete scheme for the problem under consideration and obtain optimal order error estimates.

\section{Notations and Assumptions}

Let $X$ be a Banach space with a norm $\|\cdot\|_{X}$. We denote by $X^{*}$ its dual space and by $\langle\cdot, \cdot\rangle$ the duality paring between $X^{*}$ and $X$. Let $\varphi: X \rightarrow \mathbb{R}$ be a locally Lipschitz function. The generalised directional derivative of $\varphi$ in the sense of Clarke at the point $x \in X$ in the direction $v \in X$ is defined by

$$
\varphi^{0}(x ; v):=\limsup _{y \rightarrow x, \lambda \downarrow 0} \frac{\varphi(y+\lambda v)-\varphi(y)}{\lambda} .
$$


The set of all $\zeta \in X^{*}$ such that $\varphi^{0}(x ; v) \geq\langle\zeta, v\rangle$ for all $v \in X$ is called the Clarke subdifferential (or generalised gradient) of $\varphi$ at $x \in X$ and is denoted by $\partial \varphi(x)$. Furthermore, we recall that if $\varphi: X \rightarrow \mathbb{R}$ is a locally Lipschitz function such that for all $v \in X$ the directional derivative $\varphi^{\prime}(x ; v)$ exists and $\varphi^{\prime}(x ; v)=\varphi^{0}(x ; v)$, then $\varphi$ is regular in the sense of Clarke at $x \in X$. A function $\varphi$ is said to be regular in the sense of Clarke on $X$ if it is regular at every point $x \in X$. For the properties of the generalised directional derivative, generalised gradient, and basic calculus rules cf. $[4,13]$.

Let $\Omega$ be an open bounded connected subset of $\mathbb{R}^{d}$ with the Lipschitz continuous boundary $\Gamma$, so that the unit outward normal vector $v$ exists a.e. on $\Gamma$. We also suppose that $\Gamma$ consists of three measurable and mutually disjoint parts $\bar{\Gamma}_{D}, \bar{\Gamma}_{N}$ and $\bar{\Gamma}_{N}$ such that meas $\left(\Gamma_{D}\right)>0$. We will consider the function spaces

$$
\begin{array}{ll}
H=L^{2}\left(\Omega ; \mathbb{R}^{d}\right), & \mathscr{H}=L^{2}\left(\Omega ; \mathbb{S}^{d}\right), \\
H_{1}=H^{1}\left(\Omega ; \mathbb{R}^{d}\right), & \mathscr{H}_{1}=\{\tau \in \mathscr{H} \mid \operatorname{Div} \tau \in H\}
\end{array}
$$

and introduce an additional space — viz.

$$
V=\left\{\boldsymbol{v} \in H^{1}\left(\Omega ; \mathbb{R}^{d}\right) \mid \boldsymbol{v}=0 \text { on } \Gamma_{D}\right\}
$$

needed in the variational inequality formulation of Problem 1.1. This is a Hilbert space endowed with the inner product $\langle\boldsymbol{u}, \boldsymbol{v}\rangle_{V}=\langle\boldsymbol{\varepsilon}(\boldsymbol{u}), \boldsymbol{\varepsilon}(\boldsymbol{v})\rangle_{\mathscr{H}}$ and the norm $\|\boldsymbol{u}\|_{V}=\|\boldsymbol{\varepsilon}(\boldsymbol{u})\|_{\mathscr{H}}$. The Korn inequality yields that the norms $\|\cdot\|_{V}$ and $\|\cdot\|_{H_{1}}$ are equivalent. Further, let $c_{e}$ denote the embedding constant of $V$ into $H^{\rho}\left(\Omega ; \mathbb{R}^{d}\right)$ with $\rho \in(1 / 2,1)$ and $\|\gamma\|$ the norm of the trace in $\mathscr{L}\left(H^{\rho}\left(\Omega ; \mathbb{R}^{d}\right), L^{2}\left(\Gamma ; \mathbb{R}^{d}\right)\right)$. For any $\boldsymbol{v} \in H^{1}\left(\Omega ; \mathbb{R}^{d}\right)$, the trace of $\boldsymbol{v}$ on the boundary $\Gamma$ is denoted by the same symbol $v$.

If $\xi \in \mathbb{R}^{d}$, then $\xi_{v}=\boldsymbol{\xi} \cdot \boldsymbol{v}$ and $\boldsymbol{\xi}_{\tau}=\boldsymbol{\xi}-\xi_{v} \boldsymbol{v}$ are, respectively, the normal and tangential components of $\xi$ on $\Gamma$. Analogously, the normal $(\sigma v) \cdot v$ and tangential $\sigma v-\sigma_{v} v$ components of a tensor $\boldsymbol{\sigma}$ on $\Gamma$ are respectively denoted by $\sigma_{\nu}$ and $\boldsymbol{\sigma}_{\tau}$. We note that here and in what follows the notation "." and ": " is reserved for the inner products in spaces $\mathbb{R}^{d}$ and $\mathbb{S}^{d}$, respectively.

In order to study Problem 1.1, we will make a few assumptions. Note that we use the abbreviation a.a. for almost all and a.e. for almost everywhere.

$\underline{H(\mathscr{A})}$. The viscosity operator $\mathscr{A}: Q \times \mathbb{S}^{d} \rightarrow \mathbb{S}^{d}$ satisfies the following conditions:

(a) $\mathscr{A}(\cdot, \cdot, \boldsymbol{\varepsilon})$ is measurable on $Q$ for all $\varepsilon \in \mathbb{S}^{d}$,

(b) $\mathscr{A}(x, \cdot, \boldsymbol{\varepsilon})$ is continuous on $[0, T]$ for a.a. $x \in \Omega$ and for all $\boldsymbol{\varepsilon} \in \mathbb{S}^{d}$,

(c) $\left\|\mathscr{A}\left(\boldsymbol{x}, t, \boldsymbol{\varepsilon}_{1}\right)-\mathscr{A}\left(\boldsymbol{x}, t, \boldsymbol{\varepsilon}_{2}\right)\right\|_{\mathbb{S}^{d}} \leq L_{\mathscr{A}}\left\|\varepsilon_{1}-\boldsymbol{\varepsilon}_{2}\right\|_{\mathbb{S}^{d}}$ for all $\boldsymbol{\varepsilon}_{1}, \boldsymbol{\varepsilon}_{2} \in \mathbb{S}^{d}$ and for a.a. $(\boldsymbol{x}, t) \in$ $Q$ with $L_{\mathscr{A}}>0$,

(d) $\mathscr{A}(\boldsymbol{x}, t, 0)=\mathbf{0}$ for a.a. $(\boldsymbol{x}, t) \in Q$,

(e) $\left(\mathscr{A}\left(\boldsymbol{x}, t, \varepsilon_{1}\right)-\mathscr{A}\left(\boldsymbol{x}, t, \boldsymbol{\varepsilon}_{2}\right)\right):\left(\boldsymbol{\varepsilon}_{1}-\boldsymbol{\varepsilon}_{2}\right) \geq m_{\mathscr{A}}\left\|\boldsymbol{\varepsilon}_{1}-\boldsymbol{\varepsilon}_{2}\right\|_{\mathbb{S}^{d}}^{2}$ for all $\boldsymbol{\varepsilon}_{1}, \boldsymbol{\varepsilon}_{2} \in \mathbb{S}^{d}$ and for a.a. $(x, t) \in Q$ with $m_{\mathscr{A}}>0$. 
$\underline{H(\mathscr{R})}$. The relaxation operator $\mathscr{R}: Q \times \mathbb{S}^{d} \rightarrow \mathbb{S}^{d}$ satisfies the following conditions:

(a) $\mathscr{R}(\boldsymbol{x}, t, \boldsymbol{\varepsilon})=\left(R_{i j k l}(\boldsymbol{x}, t) \varepsilon_{k l}\right)$ for all $\boldsymbol{\varepsilon}=\left(\varepsilon_{i j}\right) \in \mathbb{S}^{d}$ and for a.a. $(\boldsymbol{x}, t) \in Q$,

(b) $R_{i j k l}(\cdot, t)=R_{j i k l}(\cdot, t)=R_{l k i j}(\cdot, t)$ for all $i, j, k, l=1, \ldots, d$ and for a.a. $t \in(0, T)$,

(c) $R_{i j k l} \in C\left(0, T ; L^{\infty}(\Omega)\right)$ for all $i, j, k, l=1, \ldots, d$.

$\underline{H(p)}$. The normal function $p_{v}: \Sigma_{C} \times[0,1] \times \mathbb{R} \rightarrow \mathbb{R}$ has the following properties:

(a) $p_{v}(\cdot, r, s)$ is measurable on $\Gamma_{C}$ for all $r \in[0,1], s \in \mathbb{R}$,

(b) $\left|p_{v}\left(\boldsymbol{x}, r_{1}, s_{1}\right)-p\left(\boldsymbol{x}, r_{2}, s_{2}\right)\right| \leq m_{v}\left(\left|r_{1}-r_{2}\right|+\left|s_{1}-s_{2}\right|\right)$ for all $r_{1}, r_{2} \in[0,1], s_{1}, s_{2} \in \mathbb{R}$ and for a.a. $x \in \Sigma_{C}$ with $m_{v}>0$,

(c) $p_{\nu}(\cdot, r, s) \in L^{\infty}\left(\Gamma_{C}\right)$ for all $r \in[0,1]$ and $s \in \mathbb{R}$,

(d) $p_{v}(x, r, 0)=0$ for all $r \in[0,1]$ and for a.a. $x \in \Sigma_{C}$. tions:

$\underline{H\left(j_{\tau}\right)}$. The frictional potential $j_{\tau}: \Sigma_{C} \times[0,1] \times \mathbb{R}^{d} \rightarrow \mathbb{R}$ satisfies the following condi-

(a) $j_{\tau}(\cdot, r, \xi)$ is measurable on $\Sigma_{C}$ for all $r \in[0,1], \xi \in \mathbb{R}^{d}$ and there exists $e \in L^{2}\left(\Gamma_{C} ; \mathbb{R}^{d}\right)$ such that $j_{\tau}(\cdot, \cdot, e(\cdot)) \in L^{1}\left(\Sigma_{C}\right)$,

(b) $j_{\tau}(\boldsymbol{x}, r, \cdot)$ is locally Lipschitz on $\mathbb{R}^{d}$ for all $r \in[0,1]$ and for a.a. $(\boldsymbol{x}, t) \in \Sigma_{C}$,

(c) $\left\|\partial j_{\tau}(\boldsymbol{x}, r, \xi)\right\|_{\mathbb{R}^{d}} \leq c_{0 \tau}+c_{1 \tau}\left(|r|+\|\xi\|_{\mathbb{R}^{d}}\right)$ for all $r \in[0,1]$ and for a.a. $(\boldsymbol{x}, t) \in \Sigma_{C}$ with $c_{0 \tau}, c_{1 \tau}>0$,

(d) $j_{\tau}^{0}\left(\boldsymbol{x}, r_{1}, \boldsymbol{\xi}_{1} ; \boldsymbol{\xi}_{2}-\boldsymbol{\xi}_{1}\right)+j_{\tau}^{0}\left(\boldsymbol{x}, r_{2}, \boldsymbol{\xi}_{2} ; \boldsymbol{\xi}_{1}-\boldsymbol{\xi}_{2}\right) \leq m_{\tau}\left(\left|r_{1}-r_{2}\right|+\left\|\xi_{1}-\xi_{2}\right\|_{\mathbb{R}^{d}}\right)\left\|\xi_{1}-\xi_{2}\right\|_{\mathbb{R}^{d}}$ for all $r_{1}, r_{2} \in[0,1], \xi_{1}, \xi_{2} \in \mathbb{R}^{d}$ with $m_{\tau}>0$ and for a.a. $(x, t) \in \Sigma_{C}$.

$H(f)$. The volume forces, traction densities and the initial displacement satisfy the conditions $f_{0} \in C(0, T ; H), f_{N} \in C\left(0, T ; L^{2}\left(\Gamma_{N} ; \mathbb{R}^{d}\right)\right)$.

$H(F)$. The adhesive evolution rate function $F: \Gamma_{C} \times \mathbb{R} \times \mathbb{R}^{d} \times \mathbb{R} \rightarrow \mathbb{R}$ satisfies the following conditions:

(a) $F(\cdot, \zeta, \xi, r)$ is measurable on $\Gamma_{C}$ for all $(\zeta, \xi, r) \in \mathbb{R} \times \mathbb{R}^{d} \times \mathbb{R}$,

(b) $\left|F\left(\boldsymbol{x}, \zeta_{1}, \xi_{1}, r_{1}\right)-F\left(\boldsymbol{x}, \zeta_{2}, \boldsymbol{\xi}_{2}, r_{2}\right)\right| \leq L_{F}\left(\left|\zeta_{1}-\zeta_{2}\right|+\left\|\xi_{1}-\xi_{2}\right\|_{\mathbb{R}^{d}}+\left|r_{1}-r_{2}\right|\right)$ for all $\zeta_{i} \in \mathbb{R}$, $\xi_{i} \in \mathbb{R}^{d}, r_{i} \in \mathbb{R}, i=1,2$ and for a.a. $x \in \Gamma_{C}$,

(c) $F(x, \zeta, \xi, 0)=0, F(x, \zeta, \xi, r) \geq 0$ for $r \leq 0$ and $F(x, \zeta, \xi, r) \leq 0$ for $r \geq 1$, for all $(\zeta, \xi, r) \in \mathbb{R} \times \mathbb{R}^{d} \times \mathbb{R}$ and for a.a. $x \in \Gamma_{C}$. 
Finally, the initial bonding field and the gap function satisfy

$$
\beta_{0} \in L^{2}\left(\Gamma_{C}\right), \quad g \in L^{\infty}\left(\Gamma_{C}\right), \quad 0 \leq \beta_{0} \leq 1, \quad g \geq 0 \quad \text { a.e. on } \Gamma_{C} .
$$

In order to recall the variational formulation of Problem 1.1, we define the function $f:(0, T) \rightarrow V^{*}$ by

$$
\langle f(t), \boldsymbol{v}\rangle=\left\langle f_{0}(t), \boldsymbol{v}\right\rangle_{H}+\left\langle f_{N}(t), \boldsymbol{v}\right\rangle_{L^{2}\left(\Gamma_{N} ; \mathbb{R}^{d}\right)}
$$

for all $v \in V$ and for a.a. $t \in(0, T)$ and a closed convex subset $K$ of admissible displacements by

$$
K=\left\{\boldsymbol{v} \in V \mid v_{v} \leq g \text {, a.e. on } \Gamma_{C}\right\} .
$$

Standard arguments - cf. [13], lead to the following variational formulation of Problem 1.1.

Problem 2.1. Find a displacement field $\boldsymbol{u}:[0, T] \rightarrow V$ and a bonding field $\beta:[0, T] \rightarrow$ $L^{2}\left(\Gamma_{C}\right)$ such that $\boldsymbol{u}(t) \in K$ for all $t \in[0, T]$ and

$$
\begin{aligned}
& \left\langle\mathscr{A}(t, \boldsymbol{\varepsilon}(\boldsymbol{u}(t)), \boldsymbol{\varepsilon}(\boldsymbol{v}-\boldsymbol{u}(t))\rangle+\left\langle\int_{0}^{t} \mathscr{R}(t-s, \boldsymbol{\varepsilon}(\boldsymbol{u}(s))) d s, \boldsymbol{\varepsilon}(\boldsymbol{v}-\boldsymbol{u}(t))\right\rangle\right. \\
& +\int_{\Gamma_{C}} p_{\nu}\left(\beta(t), u_{v}(t)\right)\left(v_{v}-u_{v}(t)\right) d \Gamma+\int_{\Gamma_{C}} j_{\tau}^{0}\left(\beta(t), \boldsymbol{u}_{\tau}(t) ; \boldsymbol{v}_{\tau}-\boldsymbol{u}_{\tau}(t)\right) d \Gamma \\
\geq & \langle\boldsymbol{f}(t), \boldsymbol{v}-\boldsymbol{u}(t)\rangle \quad \text { for all } \quad \boldsymbol{v} \in K \quad \text { and } \quad t \in[0, T], \\
& \dot{\beta}(t)=F\left(u_{v}(t), \boldsymbol{u}_{\tau}(t), \beta(t)\right), \quad \beta(0)=\beta_{0} \quad \text { on } \Gamma_{C} .
\end{aligned}
$$

For the bonding field $\beta$ we will use the set $\mathscr{Q}$ defined by

$$
\mathscr{Q}=\left\{\theta:[0, T] \rightarrow L^{2}\left(\Gamma_{C}\right) \mid 0 \leq \theta(t) \leq 1, t \in[0, T] \text { a.e. on } \Gamma_{C}\right\} .
$$

Problem 2.1 includes a variational-hemivariational inequality (2.2) and a system of ordinary differential equations (2.3) with initial values on the boundary $\Gamma_{C}$. The conditions of the existence and uniqueness of solutions of Problem 2.1 are given by the following theorem.

Theorem 2.2 (cf. Han et al. [7, Theorem 5.1]). Assume that the conditions $H(\mathscr{A}), H(\mathscr{R})$, $H(F), H(p), H\left(j_{\tau}\right), H(f)$ and (2.1) hold and

$$
m_{\mathscr{A}}>\max \left\{\sqrt{3} c_{1 \tau}, m_{\tau}\right\} c_{e}^{2}\|\gamma\|^{2}+m_{\nu} c_{e}^{2}\|\gamma\|^{2} .
$$

Then Problem 2.1 has at least one solution such that

$$
\boldsymbol{u} \in C(0, T ; V) \text { and } \beta \in W^{1, \infty}\left(0, T ; L^{2}\left(\Gamma_{C}\right)\right) \cap \mathscr{Q} .
$$

Moreover, if either $j_{\tau}(x, r, \cdot)$ or $\left(-j_{\tau}(x, r, \cdot)\right)$ is regular on $\mathbb{R}^{d}$ for all $r \in \mathbb{R}$ and for a.a. $x \in \Gamma_{C}$, then the solution of Problem 2.1 is unique. 


\section{Spatially Semidiscrete Numerical Approximation}

In this section we introduce a spatially semidiscrete scheme for the variational-hemivariational inequality in Problem 2.1 and provide an error estimate. Let us first recall the Gronwall inequality and its discrete version.

Lemma 3.1 (cf. Han \& Sofonea [8, Lemma 7.24]). If $g$ is nondecreasing function and $f, g$ belong to the space $C[a, b]$ and satisfy the inequality

$$
f(t) \leq g(t)+c \int_{a}^{t} f(s) d s, \quad t \in[a, b]
$$

with a constant $c>0$, then the following estimate holds:

$$
f(t) \leq g(t) e^{c(t-a)}, \quad t \in[a, b] .
$$

Lemma 3.2 (cf. Han \& Sofonea [8, Lemma 7.26]). For a fixed $T>0$, let $N$ be a positive integer and $k:=T / N$. If $\left\{g_{n}\right\}_{n=1}^{N}$ and $\left\{e_{n}\right\}_{n=1}^{N}$ are sequences of nonnegative numbers such that

$$
e_{n} \leq \bar{c} g_{n}+\bar{c} \sum_{j=1}^{n} k e_{j}, \quad n=1, \ldots, N
$$

with a positive constant $\bar{c}$ independent of $N$ or $k$, then there exists a positive constant $c$ such that for $k$ sufficiently small, we have

$$
\max _{1 \leq n \leq N} e_{n} \leq c \max _{1 \leq n \leq N} g_{n}
$$

To simplify the notation, we will write $B$ for $L^{2}\left(\Gamma_{C}\right)$ and $(\cdot, \cdot)_{B}$ and $\|\cdot\|_{B}$ for the correspondent inner product and norm. Let $\left\{\mathscr{T}_{h}\right\}$ be a regular family of finite element partitions and $V^{h}$ the finite element space that matches the partition $\left\{\mathscr{T}_{h}\right\}$, where $h>0$ is the spatial discretisation parameter. Besides, we set

$$
K^{h}:=\left\{v^{h} \in V^{h} \mid v_{v}^{h} \leq g \text { at the node points of } \Gamma_{C}\right\} .
$$

We consider the case $K^{h} \subset K$ corresponding to the internal approximation. Let $B^{h}$ be a finite dimensional subspace of $B$ and $\mathscr{P}_{B^{h}}: B \rightarrow B^{h}$ the orthogonal projection operator defined by

$$
\left(\mathscr{P}_{B^{h}} b, b^{h}\right)_{B}=\left(b, b^{h}\right)_{B}, \quad \forall b \in B, \quad b^{h} \in B^{h} .
$$

By $\beta_{0}^{h}$ we denote the orthogonal projection of $\beta_{0}$ on $B^{h}$ and consider the following semidiscrete approximation of Problem 2.1.

Problem 3.1. Find a displacement field $\boldsymbol{u}^{h}:[0, T] \rightarrow V^{h}$ and a bonding field $\beta^{h}:[0, T] \rightarrow$ $B^{h}$ such that $\boldsymbol{u}^{h}(t) \in K^{h}$ for all $t \in[0, T]$ and

$$
\left\langle\mathscr{A}\left(t, \boldsymbol{\varepsilon}\left(\boldsymbol{u}^{h}(t)\right), \boldsymbol{\varepsilon}\left(\boldsymbol{v}^{h}-\boldsymbol{u}^{h}(t)\right)\right)\right\rangle+\left\langle\int_{0}^{t} \mathscr{R}\left(t-s, \boldsymbol{\varepsilon}\left(\boldsymbol{u}^{h}(s)\right)\right) d s, \boldsymbol{\varepsilon}\left(\boldsymbol{v}^{h}-\boldsymbol{u}^{h}(t)\right)\right\rangle
$$




$$
\begin{aligned}
& +\int_{\Gamma_{C}} p_{v}\left(\beta^{h}(t), u_{v}^{h}(t)\right)\left(v_{v}^{h}-u_{v}^{h}(t)\right) d \Gamma+\int_{\Gamma_{C}} j_{\tau}^{0}\left(\beta^{h}(t), u_{\tau}^{h}(t) ; v_{\tau}^{h}-u_{\tau}^{h}(t)\right) d \Gamma \\
\geq & \left\langle\boldsymbol{f}_{n}, \boldsymbol{v}^{h}-\boldsymbol{u}^{h}(t)\right\rangle \quad \text { for all } \quad \boldsymbol{v}^{h} \in K^{h} \text { and } t \in[0, T], \\
& \dot{\beta}^{h}(t)=\mathscr{P}_{B^{h}}\left(F\left(u_{v}^{h}(t), \boldsymbol{u}_{\tau}^{h}(t), \beta^{h}(t)\right)\right), \quad \beta^{h}(0)=\beta_{0}^{h} \quad \text { on } \Gamma_{C} .
\end{aligned}
$$

Assumption $H(\mathscr{A})(e)$ yields

$$
\begin{aligned}
& m_{\mathscr{A}}\left\|\boldsymbol{u}-\boldsymbol{u}^{h}\right\|^{2} \\
\leq & \left\langle\mathscr{A} \boldsymbol{\varepsilon}(\boldsymbol{u}(t))-\mathscr{A} \boldsymbol{\varepsilon}\left(\boldsymbol{u}^{h}(t)\right), \boldsymbol{\varepsilon}\left(\boldsymbol{u}(t)-\boldsymbol{u}^{h}(t)\right)\right\rangle \\
= & \left\langle\mathscr{A} \boldsymbol{\varepsilon}\left(\boldsymbol{u}(t)-\mathscr{A} \boldsymbol{\varepsilon}\left(\boldsymbol{u}^{h}(t)\right), \boldsymbol{\varepsilon}\left(\boldsymbol{u}(t)-\boldsymbol{v}^{h}(t)\right)\right\rangle\right. \\
& +\left\langle\mathscr{A} \boldsymbol{\varepsilon}(\boldsymbol{u}(t)), \boldsymbol{\varepsilon}\left(\boldsymbol{v}^{h}(t)-\boldsymbol{u}(t)\right)\right\rangle \\
& +\left\langle\mathscr{A} \boldsymbol{\varepsilon}(\boldsymbol{u}(t)), \boldsymbol{\varepsilon}\left(\boldsymbol{u}(t)-\boldsymbol{u}^{h}(t)\right)\right\rangle \\
& -\left\langle\mathscr{A} \boldsymbol{\varepsilon}\left(\boldsymbol{u}^{h}(t)\right), \boldsymbol{\varepsilon}\left(\boldsymbol{v}^{h}(t)-\boldsymbol{u}^{h}(t)\right)\right\rangle .
\end{aligned}
$$

If we set $v=u^{h}(t)$ in the inequality (2.2), then

$$
\begin{aligned}
& \left\langle\mathscr{A} \boldsymbol{\varepsilon}\left(\boldsymbol{u}(t), \boldsymbol{\varepsilon}\left(\boldsymbol{u}(t)-\boldsymbol{u}^{h}(t)\right)\right\rangle\right. \\
\leq & \left\langle\int_{0}^{t} \mathscr{R}(t-s, \boldsymbol{\varepsilon}(\boldsymbol{u}(s))) d s, \boldsymbol{\varepsilon}\left(\boldsymbol{u}^{h}(t)-\boldsymbol{u}(t)\right)\right\rangle \\
& +\int_{\Gamma_{C}} p_{\nu}\left(\beta(t), u_{v}(t)\right)\left(u_{v}^{h}-u_{v}(t)\right) d \Gamma \\
& +\int_{\Gamma_{C}} j_{\tau}^{0}\left(\beta(t), \boldsymbol{u}_{\tau}(t) ; \boldsymbol{u}_{\tau}^{h}-\boldsymbol{u}_{\tau}(t)\right) d \Gamma+\left\langle\boldsymbol{f}(t), \boldsymbol{u}(t)-\boldsymbol{u}^{h}(t)\right\rangle .
\end{aligned}
$$

Applying this inequality and (3.2) to the last two terms in (3.4), we obtain

$$
\begin{aligned}
m_{\mathscr{A}}\left\|\boldsymbol{u}-\boldsymbol{u}^{h}\right\|^{2} \leq & \left\langle\mathscr{A} \boldsymbol{\varepsilon}(\boldsymbol{u}(t))-\mathscr{A} \boldsymbol{\varepsilon}\left(\boldsymbol{u}^{h}(t)\right), \boldsymbol{\varepsilon}\left(\boldsymbol{u}(t)-\boldsymbol{v}^{h}(t)\right)\right\rangle \\
& +I_{1}+I_{2}+I_{3}+R\left(\boldsymbol{v}^{h}, \boldsymbol{u}\right),
\end{aligned}
$$

where

$$
\begin{aligned}
R\left(\boldsymbol{v}^{h}, \boldsymbol{u}\right)= & \left\langle\int_{0}^{t} \mathscr{R}(t-s, \boldsymbol{\varepsilon}(\boldsymbol{u}(s))) d s, \boldsymbol{\varepsilon}\left(\boldsymbol{v}^{h}(t)-\boldsymbol{u}(t)\right)\right\rangle \\
& +\int_{\Gamma_{C}} p_{v}\left(\beta(t), u_{v}(t)\right)\left(v_{v}^{h}-u_{v}(t)\right) d \Gamma \\
& +\left\langle\boldsymbol{f}(t), \boldsymbol{u}(t)-\boldsymbol{v}^{h}(t)\right\rangle+\left\langle\mathscr{A} \boldsymbol{\varepsilon}(\boldsymbol{u}(t)), \boldsymbol{\varepsilon}\left(\boldsymbol{v}^{h}(t)-\boldsymbol{u}(t)\right)\right\rangle
\end{aligned}
$$

and

$$
\begin{aligned}
& I_{1}=\left\langle\int_{0}^{t} \mathscr{R}\left(t-s, \boldsymbol{\varepsilon}\left(\boldsymbol{u}(s)-\boldsymbol{u}^{h}(s)\right)\right) d s, \boldsymbol{\varepsilon}\left(\boldsymbol{u}^{h}(t)-v^{h}\right)\right\rangle, \\
& I_{2}=\int_{\Gamma_{C}}\left(p_{v}\left(\beta(t), u_{v}(t)\right)-p_{v}\left(\beta^{h}(t), u_{v}^{h}(t)\right)\right)\left(u_{v}^{h}(t)-v_{v}^{h}\right) d \Gamma,
\end{aligned}
$$




$$
I_{3}=\int_{\Gamma_{C}} j_{\tau}^{0}\left(\beta(t), \boldsymbol{u}_{\tau}(t) ; \boldsymbol{u}_{\tau}^{h}(t)-\boldsymbol{u}_{\tau}(t)\right) d \Gamma+\int_{\Gamma_{C}} j_{\tau}^{0}\left(\beta^{h}(t), \boldsymbol{u}_{\tau}^{h}(t) ; \boldsymbol{v}_{\tau}^{h}-\boldsymbol{u}_{\tau}^{h}(t)\right) d \Gamma .
$$

In what follows, we denote by $c$ a generic positive constant independent of $h$ and $k$ whose values may be different on different occasions. The Lipschitz continuity of the operator $\mathscr{A}$ implies that

$$
\begin{aligned}
& \left\langle\mathscr{A} \boldsymbol{\varepsilon}(\boldsymbol{u}(t))-\mathscr{A} \boldsymbol{\varepsilon}\left(\boldsymbol{u}^{h}(t)\right), \boldsymbol{\varepsilon}\left(\boldsymbol{u}(t)-\boldsymbol{v}^{h}(t)\right)\right\rangle \\
\leq & L_{\mathscr{A}}\left\|\boldsymbol{u}(t)-\boldsymbol{u}^{h}(t)\right\|_{V} \cdot\left\|\boldsymbol{u}(t)-\boldsymbol{v}^{h}(t)\right\|_{V} \\
\leq & \epsilon\left\|\boldsymbol{u}(t)-\boldsymbol{u}^{h}(t)\right\|_{V}^{2}+\frac{L_{\mathscr{A}}}{4 \epsilon}\left\|\boldsymbol{u}(t)-\boldsymbol{v}^{h}(t)\right\|_{V}^{2} .
\end{aligned}
$$

In order to estimate $I_{1}$, we employ the assumption $H(\mathscr{R})$, so that

$$
I_{1} \leq T \cdot\|\mathscr{R}\|_{C\left(0, T: L^{\infty}\right)} \cdot \int_{0}^{t}\left\|\boldsymbol{u}(s)-\boldsymbol{u}^{h}(s)\right\|_{V} d s \cdot\left\|\boldsymbol{v}^{h}-\boldsymbol{u}^{h}(t)\right\|_{V} .
$$

The Cauchy-Schwarz inequality and assumption $H\left(p_{v}\right)(b)$ applied to the integral $I_{2}$ show that

$$
\begin{aligned}
I_{2} & \leq \int_{\Gamma_{C}} m_{v}\left(\left|\beta(t)-\beta^{h}(t)\right|+\left|u_{v}(t)-u_{v}^{h}(t)\right|\right) \cdot\left|v_{v}^{h}-u_{v}^{h}(t)\right| d \Gamma \\
& \leq m_{v}\left(\left\|\beta(t)-\beta^{h}(t)\right\|_{B}+\left\|u_{v}(t)-u_{v}^{h}(t)\right\|_{B}\right) \cdot\left\|v_{v}^{h}-u_{v}^{h}(t)\right\|_{B} \\
& \leq m_{v} c_{e}\|\gamma\|\left(\left\|\beta(t)-\beta^{h}(t)\right\|_{B}+c_{e}\|\gamma\|\left\|\boldsymbol{u}(t)-\boldsymbol{u}^{h}(t)\right\|_{V}\right) \cdot\left\|v^{h}-\boldsymbol{u}^{h}(t)\right\|_{V}
\end{aligned}
$$

and recalling the inequalities

$$
\begin{aligned}
& \left\|\boldsymbol{v}^{h}-\boldsymbol{u}^{h}(t)\right\|_{V} \leq\left\|\boldsymbol{u}(t)-\boldsymbol{u}^{h}(t)\right\|_{V}+\left\|\boldsymbol{v}^{h}-\boldsymbol{u}(t)\right\|_{V}, \\
& \left\|\boldsymbol{v}^{h}-\boldsymbol{u}^{h}(t)\right\|_{V}^{2} \leq 2\left(\left\|\boldsymbol{u}(t)-\boldsymbol{u}^{h}(t)\right\|_{V}^{2}+\left\|\boldsymbol{v}^{h}-\boldsymbol{u}(t)\right\|_{V}^{2}\right), \\
& a b \leq \epsilon a^{2}+\frac{1}{4 \epsilon} b^{2}, \quad \forall \epsilon>0,
\end{aligned}
$$

we finally estimate $I_{1}$ and $I_{2}$ as

$$
\begin{aligned}
& I_{1} \leq c\left(\int_{0}^{t}\left\|\boldsymbol{u}(s)-\boldsymbol{u}^{h}(s)\right\|_{V} d s\right)^{2}+c\left\|\boldsymbol{v}^{h}-\boldsymbol{u}(t)\right\|_{V}^{2}+\epsilon\left\|\boldsymbol{u}(t)-\boldsymbol{u}^{h}(t)\right\|_{V}^{2}, \\
& I_{2} \leq\left(m_{v} c_{e}^{2}\|\gamma\|^{2}+\epsilon\right)\left\|\boldsymbol{u}(t)-\boldsymbol{u}^{h}(t)\right\|_{V}^{2}+c\left(\left\|\beta(t)-\beta^{h}(t)\right\|_{B}^{2}+\left\|\boldsymbol{v}^{h}-\boldsymbol{u}(t)\right\|_{V}^{2}\right) .
\end{aligned}
$$

If, in addition, $j_{\tau}$ is Lipschitz continuous with a constant $L_{j}$ uniformly with respect to $x$ and $r$, then

$$
j_{\tau}^{0}(x, r, \xi ; \boldsymbol{\eta}) \leq L_{j}\|\boldsymbol{\eta}\|_{\mathbb{R}^{d}}
$$

for all $\xi, \eta \in \mathbb{R}^{d}$, so that $H\left(j_{\tau}\right)(d)$ and the subadditivity of the generalised directional derivative yields

$$
I_{3} \leq \int_{\Gamma_{C}} j_{\tau}^{0}\left(\beta(t), \boldsymbol{u}_{\tau}(t) ; \boldsymbol{u}_{\tau}^{h}(t)-\boldsymbol{u}_{\tau}(t)\right) d \Gamma+\int_{\Gamma_{C}} j_{\tau}^{0}\left(\beta^{h}(t), \boldsymbol{u}_{\tau}^{h}(t) ; \boldsymbol{u}_{\tau}(t)-\boldsymbol{u}_{\tau}^{h}(t)\right) d \Gamma
$$




$$
\begin{aligned}
& +\int_{\Gamma_{C}} j_{\tau}^{0}\left(\beta^{h}(t), \boldsymbol{u}_{\tau}^{h}(t) ; \boldsymbol{v}_{\tau}^{h}-\boldsymbol{u}_{\tau}(t)\right) d \Gamma \\
\leq & m_{\tau}\left(\left\|\boldsymbol{u}_{\tau}^{h}(t)-\boldsymbol{u}_{\tau}(t)\right\|_{B} \cdot\left\|\beta(t)-\beta^{h}(t)\right\|_{B}+\left\|\boldsymbol{u}_{\tau}^{h}(t)-\boldsymbol{u}_{\tau}(t)\right\|_{B}^{2}\right) \\
& +L_{j} \sqrt{\operatorname{meas}\left(\Gamma_{C}\right)} \cdot\left\|\boldsymbol{u}(t)-\boldsymbol{v}^{h}\right\|_{B} \\
\leq & (1+\epsilon) m_{\tau} c_{e}^{2}\|\gamma\|^{2}\left\|\boldsymbol{u}^{h}(t)-\boldsymbol{u}(t)\right\|_{V}^{2}+c\left\|\boldsymbol{u}(t)-\boldsymbol{v}^{h}\right\|_{B}+c\left\|\beta(t)-\beta^{h}(t)\right\|_{B}^{2} .
\end{aligned}
$$

Let us also assume that

$$
m_{\mathscr{A}}>\left(m_{\tau}+m_{v}\right) c_{e}^{2}\|\gamma\|^{2} .
$$

Then it follows from (3.5)-(3.9) that

$$
\begin{aligned}
\left\|\boldsymbol{u}(t)-\boldsymbol{u}^{h}(t)\right\|_{V}^{2} \leq & c\left(\left\|\beta(t)-\beta^{h}(t)\right\|_{B}^{2}+\left\|\boldsymbol{u}(t)-\boldsymbol{v}^{h}\right\|_{V}^{2}+\left(\int_{0}^{t}\left\|\boldsymbol{u}(s)-\boldsymbol{u}^{h}(s)\right\|_{V} d s\right)^{2}\right. \\
& \left.+\left\|\boldsymbol{u}(t)-\boldsymbol{v}^{h}\right\|_{B}+\left|R\left(\boldsymbol{v}^{h}, \boldsymbol{u}\right)\right|\right) .
\end{aligned}
$$

Therefore,

$$
\begin{aligned}
\left\|\boldsymbol{u}(t)-\boldsymbol{u}^{h}(t)\right\|_{V} \leq & c\left(\left\|\beta(t)-\beta^{h}(t)\right\|_{B}+\left\|\boldsymbol{u}(t)-\boldsymbol{v}^{h}\right\|_{V}+\int_{0}^{t}\left\|\boldsymbol{u}(s)-\boldsymbol{u}^{h}(s)\right\|_{V} d s\right. \\
& \left.+\left\|\boldsymbol{u}(t)-\boldsymbol{v}^{h}\right\|_{B}^{1 / 2}+\left|R\left(\boldsymbol{v}^{h}, \boldsymbol{u}\right)\right|^{1 / 2}\right) .
\end{aligned}
$$

We now estimate the discretisation of the adhesion evolution. Since

$$
\beta(t)-\beta^{h}(t)=\int_{0}^{t}\left(\dot{\beta}(s)-\dot{\beta}^{h}(s)\right) d s+\beta_{0}-\beta_{0}^{h},
$$

we have

$$
\dot{\beta}(s)-\dot{\beta}^{h}(s)=\left(I-\mathscr{P}_{B^{h}}\right) \dot{\beta}(s)+P_{B^{h}} \dot{\beta}(s)-\dot{\beta}^{h}(s),
$$

where $I$ is the identity operator on the space $B$. Therefore,

$$
\left\|\dot{\beta}(s)-\dot{\beta}^{h}(s)\right\|_{B} \leq\left\|\left(I-\mathscr{P}_{B^{h}}\right) \dot{\beta}(s)\right\|_{B}+\left\|P_{B^{h}} \dot{\beta}(s)-\dot{\beta}^{h}(s)\right\|_{B} .
$$

The second term on the Eq. (3.11) can be estimated by (2.3) and (3.3) — viz.

$$
\begin{aligned}
\left\|P_{B^{h}} \dot{\beta}(s)-\dot{\beta}^{h}(s)\right\|_{B} & =\left\|P_{B^{h}}\left(F\left(u_{v}(t), \boldsymbol{u}_{\tau}(t), \beta(t)\right)-F\left(u_{v}^{h}(t), \boldsymbol{u}_{\tau}^{h}(t), \beta^{h}(t)\right)\right)\right\|_{B} \\
& \leq L_{F}\left(\left|\beta(s)-\beta^{h}(s)\right|+\left|u_{v}(s)-u_{v}^{h}(s)\right|+\left\|\boldsymbol{u}_{\tau}(s)-\boldsymbol{u}_{\tau}^{h}(s)\right\|_{\mathbb{R}^{d}}\right) .
\end{aligned}
$$

It follows that

$$
\begin{aligned}
\left\|\beta(t)-\beta^{h}(t)\right\|_{B} \leq & \left\|\beta_{0}-\beta_{0}^{h}\right\|_{B}+c \int_{0}^{t}\left\|\boldsymbol{u}(s)-\boldsymbol{u}^{h}(s)\right\|_{V} d s \\
& +c \int_{0}^{t}\left(\left\|\beta(s)-\beta^{h}(s)\right\|_{B}+\left\|\left(I-\mathscr{P}_{B^{h}}\right) \dot{\beta}(s)\right\|_{B}\right) d s .
\end{aligned}
$$


Considering the error function

$$
E^{h}(t)=\left\|\beta(t)-\beta^{h}(t)\right\|_{B}+\left\|\boldsymbol{u}(t)-\boldsymbol{u}^{h}(t)\right\|_{V}
$$

and using (3.10), (3.12) for any $\boldsymbol{v}^{h} \in K^{h}$, we obtain

$$
\begin{aligned}
E^{h}(t) \leq & c \int_{0}^{t} E^{h}(s) d s+c \int_{0}^{t}\left(\left\|\left(I-\mathscr{P}_{B^{h}}\right) \dot{\beta}(s)\right\|_{B}\right) d s+\left\|\beta_{0}-\beta_{0}^{h}\right\|_{B} \\
& +c \inf _{v^{h} \in K^{h}}\left\{\left\|\boldsymbol{u}(t)-\boldsymbol{v}^{h}\right\|_{V}+\left\|\boldsymbol{u}(t)-\boldsymbol{v}^{h}\right\|_{B}^{1 / 2}+\left|R\left(\boldsymbol{v}^{h}, \boldsymbol{u}\right)\right|^{1 / 2}\right\} .
\end{aligned}
$$

Taking into account Lemma 3.1, we arrive at the following result.

Theorem 3.2. Let $(\boldsymbol{u}, \beta)$ and $\left(\boldsymbol{u}^{h}, \beta^{h}\right)$ be the solutions of Problems 2.1 and 3.1, respectively. Assume that $K^{h} \subset K, H(\mathscr{A}), H(\mathscr{R}), H(F), H(p), H\left(j_{\tau}\right), H(f)$ and the initial condition (2.1) holds. If $j_{\tau}(\boldsymbol{x}, r, \cdot)$ is Lipschitz continuous and $m_{\mathscr{A}}>\left(m_{\tau}+m_{v}\right) c_{e}^{2}\|\gamma\|^{2}$, then

$$
\begin{aligned}
\max _{0 \leq t \leq T} E^{h}(t) \leq & c \int_{0}^{T}\left\|\left(I-\mathscr{P}_{B^{h}}\right) \dot{\beta}(s)\right\|_{B} d s+c\left\|\beta_{0}-\beta_{0}^{h}\right\|_{B} \\
& +c \max _{0 \leq t \leq T}\left(\inf _{v^{h} \in K^{h}}\left\{\left\|\boldsymbol{u}(t)-\boldsymbol{v}^{h}\right\|_{V}+\left\|\boldsymbol{u}(t)-\boldsymbol{v}^{h}\right\|_{B}^{1 / 2}+\left|R\left(\boldsymbol{v}^{h}, \boldsymbol{u}\right)\right|^{1 / 2}\right\}\right) .
\end{aligned}
$$

In applications, $V^{h}$ and $B^{h}$ are often chosen as the spaces of linear and piecewise constant functions, respectively. We also assume for the moment that $\Omega$ is a polygonal or polyhedral domain and let $\mathscr{T}^{h}$ be a regular family of finite element triangulations of $\bar{\Omega}$ into triangles or tetrahedrons. For an element $T \in \mathscr{T}^{h}$, let $P_{1}\left(T ; \mathbb{R}^{d}\right)$ refer to the space of all polynomials of degree at most one on $T$. Now we consider the linear space of all piecewise continuous affine functions

$$
V^{h}=\left\{\boldsymbol{v}^{h} \in C\left(\bar{\Omega} ; \mathbb{R}^{d}\right):\left.\boldsymbol{v}^{h}\right|_{T} \in P_{1}\left(T ; \mathbb{R}^{d}\right) \text { for all } T \in \mathscr{T}^{h}, \boldsymbol{v}^{h}=\mathbf{0} \text { on } \Gamma_{D}\right\} .
$$

Recalling that $K^{h}$ is defined by (3.1), we note that if the gap function $g$ is concave, then $K^{h} \subset K$. If $\mathscr{T}_{\Gamma_{C}}^{h}$ is the partition of $\Gamma_{C}$ induced by triangulation $\mathscr{T}^{h}$, then the space $B$ is approximated by

$$
B^{h}=\left\{\theta^{h} \in B:\left.\theta^{h}\right|_{\gamma} \in \mathbb{R}, \forall \gamma \in \mathscr{T}_{\Gamma_{C}}^{h}\right\} .
$$

Suppose $\Gamma_{C}$ can be represented in the form $\Gamma_{C}=\cup_{i=1}^{i_{0}} \Gamma_{C}^{(i)}$, where each $\Gamma_{C}^{(i)}$ has a constant unit outward normal vector and consider the space $\widetilde{H}^{1}\left(\Gamma_{C}\right)$ consisting of elements $\beta$ such that $\left.\beta\right|_{\Gamma_{C}^{(i)}} \in H^{1}\left(\Gamma_{C}^{(i)}\right), 1 \leq i \leq i_{0}$. We equip $\widetilde{H}^{1}\left(\Gamma_{C}\right)$ with the norm $\|\beta\|_{\widetilde{H}^{1}\left(\Gamma_{C}\right)}:=$ $\left(\sum_{i=1}^{i_{0}}\|\beta\|_{H^{1}\left(\Gamma_{C}\right)}\right)^{1 / 2}$.

Corollary 3.1. Let the assumptions of Theorem 3.2 hold, $\Omega$ be a polygonal or polyhedral domain, $\left\{V^{h}\right\}$ and $\left\{K^{h}\right\}$ be the families of linear element spaces defined respectively by (3.14) and (3.1), and $\left\{B^{h}\right\}$ the family of piecewise constant functions defined by (3.15) corresponding to a regular family of finite element triangulations of $\bar{\Omega}$ into triangles or tetrahedrons. Moreover, let 
$(\boldsymbol{u}, \beta)$ and $\left(\boldsymbol{u}^{h}, \beta^{h}\right)$ be, respectively, the solutions of Problems 2.1 and 3.1 and $\beta_{0} \in \widetilde{H}^{1}\left(\Gamma_{C}\right)$. If $\boldsymbol{u} \in C\left(0, T ; H^{2}\left(\Omega ; \mathbb{R}^{d}\right)\right), \beta \in W^{1,1}\left(0, T ; \widetilde{H}^{1}\left(\Gamma_{C}\right)\right)$ and $\boldsymbol{\sigma} \boldsymbol{v} \in C\left(0, T ; L^{2}\left(\Gamma ; \mathbb{R}^{d}\right)\right)$, then the following optimal order error estimate

$$
\max _{0 \leq t \leq T} E^{h}(t) \leq c h
$$

holds.

Proof. The regularity conditions yield that for a.a. $t \in[0, T]$, the function $\boldsymbol{u}(t)$ is continuous on $\bar{\Omega}$ and $\boldsymbol{u}_{\tau}(t)$ is continuous on $\Gamma_{C}$. Let $\boldsymbol{v}^{h}(t)=\Pi^{h} \boldsymbol{u}(t) \in K^{h}$ be the finite element interpolant of $\boldsymbol{u}(t)$ on $[0, T]$. The standard finite element interpolant error estimates $[1,3,8]$ imply the following approximation properties — cf. [1, Theorem 10.3.9]:

$$
\begin{array}{ll}
\left\|\boldsymbol{u}(t)-\Pi^{h} \boldsymbol{u}(t)\right\|_{V} \leq c h\|\boldsymbol{u}(t)\|_{H^{2}\left(\Omega ; \mathbb{R}^{d}\right)} & \text { for a.a. } t \in[0, T], \\
\left\|\boldsymbol{u}(t)-\Pi^{h} \boldsymbol{u}(t)\right\|_{B} \leq c h^{2}\|\boldsymbol{u}(t)\|_{H^{2}\left(\Gamma_{C} ; \mathbb{R}^{d}\right)} & \text { for a.a. } t \in[0, T], \\
c \int_{0}^{T}\left\|\left(I-\mathscr{P}_{B^{h}}\right) \dot{\beta}(s)\right\|_{B} d s \leq c \int_{0}^{T} h\|\dot{\beta}(s)\|_{\widetilde{H}^{1}\left(\Gamma_{C}\right)} d s \leq c h . &
\end{array}
$$

Since $\beta_{0}^{h} \in B^{h}$ is the orthogonal projection of $\beta_{0}$ on $B^{h}$ and $\beta_{0} \in \widetilde{H}^{1}\left(\Gamma_{C}\right)$, then

$$
\left\|\beta_{0}-\beta_{0}^{h}\right\| \leq c h .
$$

It remains to consider $\left|R\left(\boldsymbol{v}^{h}, \boldsymbol{u}\right)\right|$. Multiplying (1.2) by $\boldsymbol{v}-\boldsymbol{u}$ with $\boldsymbol{v} \in K$, integrating the resulting equation over $\Omega$ and using integration by parts, we obtain

$$
\int_{\Omega} \boldsymbol{\sigma} \cdot \boldsymbol{\varepsilon}(\boldsymbol{v}-\boldsymbol{u}(t)) d x=\langle\boldsymbol{f}, \boldsymbol{v}-\boldsymbol{u}(t)\rangle+\int_{\Gamma_{C}} \boldsymbol{\sigma} \boldsymbol{v} \cdot(\boldsymbol{v}-\boldsymbol{u}(t)) .
$$

Setting $v=v^{h}$ yields

$$
R\left(\boldsymbol{v}^{h}, \boldsymbol{u}\right)=\int_{\Gamma_{C}} p_{v}\left(\beta(t), u_{v}(t)\right)\left(v_{v}^{h}-u_{v}(t)\right) d \Gamma+\int_{\Gamma_{C}} \boldsymbol{\sigma} \boldsymbol{v} \cdot\left(\boldsymbol{v}^{h}-\boldsymbol{u}(t)\right) d \Gamma
$$

It follows from $H(p)(c)$ and the assumption $\boldsymbol{\sigma} v \in C\left(0, T ; L^{2}\left(\Gamma_{C} ; \mathbb{R}^{d}\right)\right)$ that

$$
\left|R\left(\boldsymbol{v}^{h}, \boldsymbol{u}\right)\right| \leq c\left\|\boldsymbol{v}^{h}-\boldsymbol{u}(t)\right\|_{B} .
$$

Applying inequalities (3.17)-(3.21) to the right-hand side of (3.13), we obtain (3.16).

\section{Fully Discrete Numerical Approximation}

To construct a fully discrete approximation of Problem 2.1, we consider the equidistant time grid $t_{n}=n k, n=0,1, \ldots, N$, with $k=T / N$. Let $\boldsymbol{u}_{0}^{h} \in V^{h}$ and $\beta_{0}^{h} \in B^{h}$ be suitable 
approximations of $\boldsymbol{u}_{0}$ and $\beta_{0}$. For a time continuous function $\phi=\phi(t)$, we write $\phi_{n}=$ $\phi\left(t_{n}\right)$ for $n=0,1, \ldots, N$.

The integral terms are approximated by the right rectangle formula on each subinterval $\left[t_{j}, t_{j+1}\right] \subset[0, T]$. Moreover, the time integral operator in Problem 2.1 is approximated as

$$
\int_{0}^{t_{n}} \mathscr{R}\left(t_{n}-s, \boldsymbol{\varepsilon}(\boldsymbol{u}(s))\right) d s \approx k \sum_{j=1}^{n} \mathscr{R}\left(t_{n}-t_{j}, \boldsymbol{\varepsilon}\left(\boldsymbol{u}_{j}\right)\right) .
$$

We now consider the following problem.

Problem 4.1. Find a discrete displacement field $\boldsymbol{u}^{h k}=\left\{\boldsymbol{u}_{n}^{h k}\right\}_{n=0}^{N} \subset K^{h}$ and a discrete bonding field $\beta^{h k}=\left\{\beta_{n}^{h k}\right\}_{n=0}^{N} \subset B^{h}$ such that $\boldsymbol{u}_{0}^{h k}=\boldsymbol{u}_{0}^{h}, \beta_{0}^{h k}=\beta_{0}^{h}$ and for $n=1,2, \ldots, N$ one has

$$
\begin{aligned}
&\left\langle\mathscr{A}\left(t_{n}, \boldsymbol{\varepsilon}\left(\boldsymbol{u}_{n}^{h k}\right)\right), \boldsymbol{\varepsilon}\left(\boldsymbol{v}^{h}-\boldsymbol{u}_{n}^{h k}\right)\right\rangle+\left\langle k \sum_{j=1}^{n} \mathscr{R}\left(t_{n}-t_{j}, \boldsymbol{\varepsilon}\left(\boldsymbol{u}_{j}^{h k}\right)\right), \boldsymbol{\varepsilon}\left(\boldsymbol{v}^{h}-\boldsymbol{u}_{n}^{h k}\right)\right\rangle \\
&+\int_{\Gamma_{C}} p_{v}\left(\beta_{n}^{h k}, u_{n, v}^{h k}\right)\left(v_{v}^{h}-u_{n, v}^{h k}\right) d \Gamma+\int_{\Gamma_{C}} j_{\tau}^{0}\left(\beta_{n}^{h k}, \boldsymbol{u}_{n, \tau}^{h k} ; \boldsymbol{v}_{\tau}^{h}-\boldsymbol{u}_{n, \tau}^{h k}\right) d \Gamma \\
& \geq\left\langle\boldsymbol{f}(t), \boldsymbol{v}^{h}-\boldsymbol{u}_{n}^{h k}\right\rangle \quad \text { for all } \quad \boldsymbol{v}^{h} \in K^{h}, \\
& \delta \beta_{n}^{h k}=\mathscr{P}_{B^{h}}\left(F\left(u_{n-1, v}^{h k}, \boldsymbol{u}_{n-1, \tau}^{h k}, \beta_{n-1}^{h k}\right)\right) \text { on } \Gamma_{C} .
\end{aligned}
$$

Let $\boldsymbol{u}_{0}^{h}$ be the finite element interpolant of $\boldsymbol{u}_{0}$ in $V^{h}$ and $\beta_{0}^{h}=\mathscr{P}_{B_{h}}\left(\beta_{0}\right)$. We now consider the error $\left\|\beta_{n}-\beta_{n}^{h k}\right\|_{B}$.

Lemma 4.1. Assume that

$$
\begin{aligned}
& \boldsymbol{u}_{0} \in H^{2}\left(\Omega ; \mathbb{R}^{d}\right), \quad \beta_{0} \in \widetilde{H}^{1}\left(\Gamma_{C}\right), \\
& u \in W^{2,1}(0, T ; V) \cap C\left([0, T] ; H^{2}(\Omega)^{d}\right), \\
& \beta \in W^{2,1}(0, T ; B) \cap C^{1}\left([0, T] ; \widetilde{H}^{1}\left(\Gamma_{C}\right)\right),
\end{aligned}
$$

and conditions $H(F)$ hold. Then

$$
\left\|\beta_{n}-\beta_{n}^{h k}\right\|_{B} \leq c k \sum_{j=1}^{n}\left(\left\|\beta_{j-1}-\beta_{j-1}^{h k}\right\|_{B}+\left\|\boldsymbol{u}_{j-1}-\boldsymbol{u}_{j-1}^{h k}\right\|_{V}\right)+c(h+k) .
$$

Proof. Exploiting the representation

$$
\beta_{n}-\beta_{n}^{h k}=\beta_{0}-\beta_{0}^{h k}+k \sum_{j=1}^{n} \delta\left(\beta_{j}-\beta_{j}^{h k}\right),
$$

we obtain

$$
\begin{aligned}
\left\|\beta_{n}-\beta_{n}^{h k}\right\|_{B} & \leq\left\|\beta_{0}-\beta_{0}^{h k}\right\|_{B}+k \sum_{i=1}^{n}\left\|\delta\left(\beta_{j}-\beta_{j}^{h k}\right)\right\|_{B} \\
& \leq\left\|\beta_{0}-\beta_{0}^{h k}\right\|_{B}+k \sum_{i=1}^{n}\left\|\delta \beta_{j}-\dot{\beta}_{j}\right\|_{B}+k \sum_{i=1}^{n}\left\|\dot{\beta}_{j}-\delta \beta_{j}^{h k}\right\|_{B} .
\end{aligned}
$$


In order to estimate $\left\|\dot{\beta}_{j}-\delta \beta_{j}^{h k}\right\|_{B}$, we apply (2.3), (4.2) and $H(F)$, thus obtaining

$$
\begin{aligned}
\left\|\dot{\beta}_{j}-\delta \beta_{j}^{h k}\right\|_{B} & =\left\|\left(I-\mathscr{P}_{B^{h}}\right) \dot{\beta}_{j}\right\|_{B}+\left\|P_{B^{h}} \dot{\beta}_{j}-\dot{\beta}_{j}^{h k}\right\|_{B} \\
& \leq\left\|\left(I-\mathscr{P}_{B^{h}}\right) \dot{\beta}_{j}\right\|_{B}+\left\|F\left(u_{j, v}, \boldsymbol{u}_{j, \tau}, \beta_{j}\right)-F\left(u_{j-1, v}^{h k}, \boldsymbol{u}_{j-1, \tau}^{h k}, \beta_{j-1}^{h k}\right)\right\|_{B} \\
& \leq\left\|\left(I-\mathscr{P}_{B^{h}}\right) \dot{\beta}_{j}\right\|_{B}+\sqrt{3} L_{F}\left(\left|\beta_{j}-\beta_{j-1}^{h k}\right|+\left|u_{j, v}-u_{j-1, v}^{h k}\right|+\left\|\boldsymbol{u}_{j, \tau}-\boldsymbol{u}_{j-1, \tau}^{h k}\right\|_{\mathbb{R}^{d}}\right) .
\end{aligned}
$$

Consequently,

$$
\left\|\dot{\beta}_{j}-\delta \beta_{j}^{h k}\right\|_{B} \leq c\left(\left\|\beta_{j}-\beta_{j-1}^{h k}\right\|_{B}+\left\|\boldsymbol{u}_{j}-\boldsymbol{u}_{j-1}^{h k}\right\|_{V}\right)+\left\|\left(I-\mathscr{P}_{B^{h}}\right) \dot{\beta}_{j}\right\|_{B} .
$$

The terms in the right-hand side of (4.7) can be estimated as follows

$$
\begin{gathered}
\left\|\boldsymbol{u}_{j}-\boldsymbol{u}_{j-1}^{h k}\right\|_{V} \leq\left\|\boldsymbol{u}_{j-1}-\boldsymbol{u}_{j-1}^{h k}\right\|_{V}+\left\|\boldsymbol{u}_{j}-\boldsymbol{u}_{j-1}\right\|_{V}, \\
\left\|\beta_{j}-\beta_{j-1}^{h k}\right\|_{B} \leq\left\|\beta_{j-1}-\beta_{j-1}^{h k}\right\|_{B}+\left\|\beta_{j}-\beta_{j-1}\right\|_{B} .
\end{gathered}
$$

It follows from the regularity assumptions (4.3), (4.4) that

$$
\begin{aligned}
& \left\|\boldsymbol{u}_{j}-\boldsymbol{u}_{j-1}\right\|_{V} \leq c k, \quad\left\|\boldsymbol{u}_{0}-\boldsymbol{u}_{0}^{h k}\right\|_{V} \leq c h, \\
& \left\|\beta_{0}-\beta_{0}^{h k}\right\|_{B} \leq c h, \quad\left\|\beta_{j}-\beta_{j-1}\right\|_{B} \leq c k, \\
& \left\|\delta \beta_{j}-\dot{\beta}_{j}\right\|_{B}=\left\|\frac{1}{k} \int_{t_{j-1}}^{t_{j}}\left(\dot{\beta}(s)-\dot{\beta}\left(t_{j}\right)\right) d s\right\|_{B} \leq\|\ddot{\beta}\|_{L^{1}\left(t_{j-1}, t_{j} ; B\right)} .
\end{aligned}
$$

Using these inequalities in (4.6), we obtain (4.5).

We now estimate $\boldsymbol{u}_{n}-\boldsymbol{u}_{n}^{\text {hk }}$. Proceeding similar to considerations for (3.5), we use (2.2) and (4.1) to obtain the inequality

$$
\begin{aligned}
m_{\mathscr{A}}\left\|\boldsymbol{u}_{n}-\boldsymbol{u}_{n}^{h k}\right\|^{2} \leq & \left\langle\mathscr{A} \boldsymbol{\varepsilon}\left(\boldsymbol{u}_{n}\right)-\mathscr{A} \boldsymbol{\varepsilon}\left(\boldsymbol{u}_{n}^{h k}\right), \boldsymbol{\varepsilon}\left(\boldsymbol{u}_{n}-\boldsymbol{v}^{h}\right)\right\rangle \\
& +I_{1}^{h k}+I_{2}^{h k}+I_{3}^{h k}+R_{n}\left(\boldsymbol{v}^{h}, \boldsymbol{u}_{n}\right),
\end{aligned}
$$

where

$$
\begin{aligned}
R_{n}\left(\boldsymbol{v}^{h}, \boldsymbol{u}_{n}\right)= & \left\langle\int_{0}^{t_{n}} \mathscr{R}\left(t_{n}-s, \boldsymbol{\varepsilon}(\boldsymbol{u}(s))\right) d s, \boldsymbol{\varepsilon}\left(\boldsymbol{v}^{h}-\boldsymbol{u}_{n}\right)\right\rangle+\int_{\Gamma_{C}} p_{v}\left(\beta_{n}, u_{n, v}\right)\left(v_{v}^{h}-u_{n, v}\right) d \Gamma \\
& +\left\langle\boldsymbol{f}_{n}, \boldsymbol{u}_{n}-\boldsymbol{v}^{h}\right\rangle+\left\langle\mathscr{A} \boldsymbol{\varepsilon}\left(\boldsymbol{u}_{n}\right), \boldsymbol{\varepsilon}\left(\boldsymbol{v}^{h}-\boldsymbol{u}_{n}\right)\right\rangle,
\end{aligned}
$$


The considerations similar to (3.8) and (3.9) show that

$$
\begin{aligned}
& \left\langle\mathscr{A} \boldsymbol{\varepsilon}\left(\boldsymbol{u}_{n}\right)-\mathscr{A} \boldsymbol{\varepsilon}\left(\boldsymbol{u}_{n}^{h k}\right), \boldsymbol{\varepsilon}\left(\boldsymbol{u}_{n}-\boldsymbol{v}^{h}\right)\right\rangle \\
\leq & L_{\mathscr{A}}\left\|\boldsymbol{u}_{n}-\boldsymbol{u}_{n}^{h k}\right\|_{V} \cdot\left\|\boldsymbol{u}_{n}-\boldsymbol{v}^{h}\right\|_{V} \leq \epsilon\left\|\boldsymbol{u}_{n}-\boldsymbol{u}_{n}^{h k}\right\|_{V}^{2}+\frac{L_{\mathscr{A}}}{4 \epsilon}\left\|\boldsymbol{u}_{n}-\boldsymbol{v}^{h}\right\|_{V}^{2}, \\
& I_{2}^{h k} \leq\left(m_{v} c_{e}^{2}\|\gamma\|^{2}+\epsilon\right)\left\|\boldsymbol{u}_{n}-\boldsymbol{u}_{n}^{h k}\right\|^{2}+c\left(\left\|\beta_{n}-\beta_{n}^{h k}\right\|_{B}^{2}+\left\|\boldsymbol{v}^{h}-\boldsymbol{u}_{n}\right\|_{V}^{2}\right), \\
& I_{3}^{h k} \leq m_{\tau} c_{e}^{2}\|\gamma\|^{2}\left\|\boldsymbol{u}_{n}^{h k}-\boldsymbol{u}_{n}\right\|_{V}^{2}+c\left\|\boldsymbol{u}_{n}-\boldsymbol{v}^{h}\right\|_{B} .
\end{aligned}
$$

Estimating $I_{1}^{h k}$, we write

$$
\begin{aligned}
& \left\|\int_{0}^{t_{n}} \mathscr{R}\left(t_{n}-s, \boldsymbol{\varepsilon}(\boldsymbol{u}(s))\right) d s-k \sum_{j=1}^{n} \mathscr{R}\left(t_{n}-t_{j}, \boldsymbol{\varepsilon}\left(\boldsymbol{u}_{j}^{h k}\right)\right)\right\|_{\mathscr{H}} \\
\leq & \left\|\int_{0}^{t_{n}} \mathscr{R}\left(t_{n}-s, \boldsymbol{\varepsilon}(\boldsymbol{u}(s))\right) d s-k \sum_{j=1}^{n} \mathscr{R}\left(t_{n}-t_{j}, \boldsymbol{\varepsilon}\left(\boldsymbol{u}_{j}\right)\right)\right\|_{\mathscr{H}} \\
& +\left\|k \sum_{j=1}^{n} \mathscr{R}\left(t_{n}-t_{j}, \boldsymbol{\varepsilon}\left(\boldsymbol{u}_{j}\right)\right)-k \sum_{j=1}^{n} \mathscr{R}\left(t_{n}-t_{j}, \boldsymbol{\varepsilon}\left(\boldsymbol{u}_{j}^{h k}\right)\right)\right\|_{\mathscr{H}} \\
\leq & c k\left\|\frac{d}{d s}\left(\mathscr{R}\left(t_{n}-s, \boldsymbol{\varepsilon}(\boldsymbol{u}(s))\right)\right)\right\|_{L^{\infty}(0, T ; \mathscr{H})}+c k \sum_{j=1}^{n}\left\|\boldsymbol{u}_{j}-\boldsymbol{u}_{j}^{h k}\right\|_{V} \\
\leq & c k\left(\|\boldsymbol{u}\|_{W^{1, \infty}(0, T ; V)}+\sum_{j=1}^{n}\left\|\boldsymbol{u}_{j}-\boldsymbol{u}_{j}^{h k}\right\|_{V}\right) .
\end{aligned}
$$

Then

$$
I_{1}^{h k} \leq c k\left\|\boldsymbol{u}_{n}^{h k}-\boldsymbol{v}^{h}\right\|_{V}\left(\|\boldsymbol{u}\|_{W^{1, \infty}(0, T ; V)}+\sum_{j=1}^{n}\left\|\boldsymbol{u}_{j}-\boldsymbol{u}_{j}^{h k}\right\|_{V}\right) .
$$

Assuming now that $m_{\mathscr{A}}>\left(m_{\tau}+m_{v}\right) c_{e}^{2}\|\gamma\|^{2}$ and following the procedure preceding the estimate (3.10), we obtain

$$
\begin{aligned}
\left\|\boldsymbol{u}_{n}-\boldsymbol{u}_{n}^{h k}\right\|_{V}^{2} \leq & c\left(\left\|\beta_{n}-\beta_{n}^{h k}\right\|_{B}^{2}+\left\|\boldsymbol{u}_{n}-\boldsymbol{v}^{h}\right\|_{V}^{2}+\left\|\boldsymbol{u}_{n}-\boldsymbol{v}^{h}\right\|_{B}+R_{n}\left(\boldsymbol{v}^{h}, \boldsymbol{u}_{n}\right)\right) \\
& +c k\left\|\boldsymbol{u}_{n}^{h k}-\boldsymbol{v}^{h}\right\|_{V}\left(\|\boldsymbol{u}\|_{W^{1, \infty}(0, T ; V)}+\sum_{j=1}^{n}\left\|\boldsymbol{u}_{j}-\boldsymbol{u}_{j}^{h k}\right\|_{V}\right) .
\end{aligned}
$$

The application of the inequalities

$$
a b \leq \epsilon a^{2}+\frac{1}{4 \epsilon} b^{2}, \quad\left\|u_{n}^{h k}-v^{h}\right\|_{V}^{2} \leq 2\left(\left\|u_{n}^{h k}-u_{n}\right\|_{V}^{2}+\left\|u_{n}-v^{h}\right\|_{V}^{2}\right)
$$

to the terms in the right-hand side of (4.8) yields

$$
\begin{aligned}
\left\|\boldsymbol{u}_{n}-\boldsymbol{u}_{n}^{h k}\right\|_{V}^{2} \leq & c\left(\left\|\beta_{n}-\beta_{n}^{h k}\right\|_{B}^{2}+\left\|\boldsymbol{u}_{n}-\boldsymbol{v}^{h}\right\|_{V}^{2}+\left\|\boldsymbol{u}_{n}-\boldsymbol{v}^{h}\right\|_{B}+\left|R_{n}\left(\boldsymbol{v}^{h}, \boldsymbol{u}_{n}\right)\right|\right) \\
& +c k\left(\|\boldsymbol{u}\|_{W^{1, \infty}(0, T ; V)}+\sum_{j=1}^{n}\left\|\boldsymbol{u}_{j}-\boldsymbol{u}_{j}^{h k}\right\|_{V}\right) .
\end{aligned}
$$


Considering the error function

$$
E_{n}^{h k}=\left\|\beta_{n}-\beta_{n}^{h k}\right\|_{B}+\left\|\boldsymbol{u}_{n}-\boldsymbol{u}_{n}^{h k}\right\|_{V}
$$

and using (4.5), (4.9), we obtain that for $v^{h} \in K^{h}$ the estimate

$$
E_{n}^{h k} \leq c \inf _{v^{h} \in K^{h}}\left\{\left\|\boldsymbol{u}_{n}-\boldsymbol{v}^{h}\right\|_{V}+\left\|\boldsymbol{u}_{n}-\boldsymbol{v}^{h}\right\|_{B}^{1 / 2}+\left|R_{n}\left(\boldsymbol{v}^{h}, \boldsymbol{u}_{n}\right)\right|^{1 / 2}\right\}+c k \sum_{j=1}^{n} E_{j}^{h k}+c(h+k)
$$

holds. Recalling Lemma 3.2, we arrive at the inequality

$$
\max _{0 \leq n \leq N} E_{n}^{h k} \leq \max _{0 \leq n \leq N}\left(\inf _{v^{h} \in K^{h}}\left\{\left\|\boldsymbol{u}_{n}-\boldsymbol{v}^{h}\right\|_{V}+\left\|\boldsymbol{u}_{n}-\boldsymbol{v}^{h}\right\|_{B}^{1 / 2}+\left|R_{n}\left(\boldsymbol{v}^{h}, \boldsymbol{u}_{n}\right)\right|^{1 / 2}\right\}\right)+c(h+k) .
$$

Thus we established the following theorem.

Theorem 4.2. Under the conditions of Theorem 3.2, Lemma 4.1 and assumptions (4.3), (4.4), we choose the finite dimensional spaces $V^{h}, K^{h}$ and $B^{h}$ as in Corollary 3.1. If ( $\left.u, \beta\right)$ and $\left(\boldsymbol{u}^{h k}, \beta^{h k}\right)$ are, respectively, the solutions of Problems 2.1 and 4.1 and $\boldsymbol{u} \in W^{1, \infty}(0, T ; V)$, then

$$
\max _{0 \leq n \leq N}\left\|\beta_{n}-\beta_{n}^{h k}\right\|_{B}+\left\|\boldsymbol{u}_{n}-\boldsymbol{u}_{n}^{h k}\right\|_{V} \leq c(h+k) .
$$

The proof is omitted here. It is similar to the proof of Corollary 3.1 and follows from (4.10), (3.17), (3.18) and (3.21).

\section{Conclusions}

We constructed spatially semidiscrete and fully discrete schemes for a variational-hemivariational inequality, which describes adhesive contact between a deformable body of a viscoelastic material with long memory and a foundation. Assuming certain regularity of the solution and using piecewise linear finite element function for displacements and piecewise constant functions for bonding field, we obtain optimal order error estimates.

\section{Acknowledgments}

The authors express their sincere gratitude to the anonymous referees for valuable comments and suggestions, which help to improve the manuscript.

This work was supported by the National Natural Science Foundation of China under Grant No. 11571311 and by the European Union's Horizon 2020 Research and Innovation Programme under the Marie Sklodowska-Curie Grand Agreement No. 823731 CONMECH. 


\section{References}

[1] K.E. Atkinson and W. Han, Theoretical numerical analysis: A functional analysis framework, Springer-Verlag (2008).

[2] K. Bartosz, Hemivariational inequalities modeling dynamic contact problems with adhesion, Nonlinear Anal. 71, 1747-1762 (2009).

[3] P.G. Ciarlet, The finite element method for elliptic problems, North-Holland (1978).

[4] F.H. Clarke, Optimization and nonsmooth analysis, Classics in applied mathematics 5, SIAM (1990).

[5] M. Cocu and R. Rocca, Existence results for unilateral quasistatic contact problems with friction and adhesion, ESAIM Math. Model. Numer. Anal. 34, 981-1001 (2000).

[6] J. Czepiel and P. Kalita, Numerical solution of a variational-hemivariational inequality modelling simplified adhesion of an elastic body, IMA J. Numer. Anal. 35, 372-393 (2015).

[7] J. Han, Y. Li and S. Migórski, Analysis of an adhesive contact problem for viscoelastic materials with long memory, J. Math. Anal. Appl. 427, 646-668 (2015).

[8] W. Han and M. Sofonea, Quasistatic contact problems in viscoelasticity and viscoplasticity, AMS/IP (2002).

[9] W. Han, M. Sofonea and M. Barboteu, Numerical analysis of elliptic hemivariational inequalities, SIAM J. Numer. Anal. 55, 640-663 (2017).

[10] J. Jarušek and M. Sofonea, On the solvability of dynamic elastic-visco-plastic contact problems, Ann. Acad. Rom. Sci. Ser. Math. Appl. 88, 3-22 (2010).

[11] S. Migórski and A. Ochal, A unified approach to dynamic contact problems in viscoelasticity, J. Elasticity. 83, 247-275 (2006).

[12] S. Migórski and A. Ochal, Dynamic bilateral contact problem for viscoelastic piezoelectric materials with adhesion, Nonlinear Anal. 69, 495-509 (2008).

[13] S. Migórski, A. Ochal and M. Sofonea, Nonlinear inclusions and hemivariational inequalities. Models and analysis of contact problems, Adv. Mech. Math. 26, Springer-Verlag (2013).

[14] Z. Naniewicz and P.D. Panagiotopoulos, Mathematical theory of hemivariational inequalities and applications, Pure Appl. Math. (Boca Raton) 188, CRC Press (1994).

[15] P.D. Panagiotopoulos, Hemivariational inequalities: Applications in mechanics and engineering, Springer-Verlag (1993).

[16] M. Raous and L. Cangémi, M. Cocu, A consistent model coupling adhesion, friction, and unilateral contact, Comput. Methods Appl. Mech. Engrg. 177, 383-399 (1999).

[17] T. Roubíček, Adhesive contact of visco-elastic bodies and defect measures arising by vanishing viscosity, SIAM J. Math. Anal. 45, 101-126 (2013) .

[18] T. Roubíček, C.G. Panagiotopoulos and V. Mantič, Quasistatic adhesive contact of visco-elastic bodies and its numerical treatment for very small viscosity, ZAMM Z. Angew. Math. Mech. 93, 823-840 (2013).

[19] M. Shillor, M. Sofonea and J.J. Telega, Models and analysis of quasistatic contact, Variational Methods. 655, Springer-Verlag (2004).

[20] M. Sofonea, W. Han and M. Shillor, Analysis and approximation of contact problems with adhesion or damage, Pure Appl. Math. (Boca Raton) 276, CRC Press (2006). 